\title{
PARÂMETROS DE CRESCIMENTO DE SPIRULINA SP. (LEB18) UTILIZADA COMO AGENTE PARA REMOÇÃO DE NUTRIENTES EM ÁGUA RESIDUAL DA AQUICULTURA
}

Ana Victória dos Santos Borges1; Lucas Guimarães Cardoso²; Jessica Hartwig Duarte $^{3}$; Jorge Alberto Vieira Costa ${ }^{3}$; Denilson de Jesus Assis; ; Janice Izabel Druzian ${ }^{5}$; Fabio Alexandre Chinalia2; Karina Lizzeth Pedraza Galván5.

${ }^{1}$ Escola Politécnica, Departamento de Engenharia Química, Universidade Federal da Bahia, Salvador/Bahia; ana.b@outlook.com.br

2 Instituto de Ciências da Saúde, Departamento de Biotecnologia, Universidade Federal da Bahia, Salvador/Bahia.

${ }^{3}$ Laboratório de Engenharia Bioquímica, Faculdade de Química e Engenharia de Alimentos, Universidade Federal do Rio Grande, Rio Grande.

${ }^{4}$ Escola de Arquitetura, Engenharia e Tecnologia da Informação, Universidade de Salvador, Salvador/Bahia.

${ }^{5}$ Programa de Graduação em Ciência dos Alimentos, Faculdade de Farmácia da Universidade Federal da Bahia, Salvador/Bahia.

Resumo: O objetivo foi produzir Spirulina sp. (LEB 18) biomassa pela reutilização e tratamento de águas residuárias da aquicultura, determinando o melhor desempenho produtivo através de parâmetros cinéticos. Os cultivos foram realizados em fotobiorreatores (1L) com $100 \%$ de água residual de aquicultura suplementada com T-25, T-50, T-75. Não houve diferença significativa na concentração de biomassa nos tratamentos $50 \%\left(1,02 \mathrm{gL}^{-1}\right), 25 \%\left(1,10 \mathrm{gL}^{-1}\right)$ e controle $\left(1,05 \mathrm{gL}^{-1}\right)$. Assim, o tratamento com $25 \%$ representa uma alternativa eficiente, barata e sustentável para o setor de aquicultura, reduzindo os impactos das descargas de efluentes, produzindo biomassa de baixo custo com características diferenciadas e alto valor agregado.

Palavras-Chave: microalga; água residual; aquicultura.

\section{GROWTH PARAMETERS OF SPIRULINA SP. (LEB18) USED AS AN AGENT FOR THE REMOVAL OF NUTRIENTS IN AQUACULTURE RESIDUAL WATER}

\begin{abstract}
The objective was to produce Spirulina sp. (LEB 18) biomass by reuse and treatment of aquaculture wastewater, determining the best productive performance through kinetic parameters. Cultures were performed in photobioreactors (1L) with $100 \%$ residual aquaculture water supplemented with T-25, T-50, T-75. There was no significant difference in biomass concentration in the 50\% $\left(1.02 \mathrm{gL}^{-1}\right), 25 \%\left(1.10 \mathrm{gL}^{-1}\right)$ and control $\left(1.05 \mathrm{gL}^{-1}\right)$ treatments. Thus, the $25 \%$ treatment represents an efficient, cheap and sustainable alternative for the aquaculture sector, reducing the impacts of effluent discharges, producing low cost biomass with different characteristics and high added value.
\end{abstract}

Keywords: microalgae; wastewater; aquaculture. 


\section{INTRODUÇÃO}

A aquicultura cresce a uma taxa constante de $4,5 \%$ ao ano. Em 2017, essa atividade industrial atingiu uma safra global total de 83,6 milhões de toneladas, representando $55 \%$ do total de peixes utilizados para consumo humano direto (FAO, 2018). Esse cultivo usa grande volume de água doce e limpa gerando águas residuais ricas em nutrientes [1].

Várias tecnologias foram desenvolvidas visando o tratamento de águas residuais da aquicultura sendo o cultivo de microalgas apontado como o tratamento contendo baixo custo, alcançando até $90 \%$ de remoção dos nutrientes orgânicos e inorgânicos com o beneficio de produzir biomassa com baixo culto e alto valor agregado. Dentre as microalgas, a Spirulina platensis ocupa uma posição de destaque no mercado global, pois trata-se de uma microalga rica em proteínas $(\sim 70 \%)$, gorduras (3-9\%), carboidratos (15 a 30\%), $\beta$-caroteno (maior que cenouras) e vitaminas (incluindo B1, B2 e B12). Por esse motivo, a biomassa da Spirulina sp. é utilizada como suplemento alimentar para seres humanos e animais [1,2].

O crescimento da indústria de biomassa da Spirulina sp. e a aquicultura dependem diretamente da implementação de novos sistemas de cultivo destinados a reduzir custos e minimizar os impactos ambientais. Esta pesquisa visa aliar a produção de biomassa de Spirulina sp. através da reutilização e tratamento de águas residuais de aquicultura, determinando o melhor desempenho de produção através de parâmetros cinéticos. Para atingir tal objetivo, o efluente aquícola foi suplementado com diferentes quantidades de nutrientes para favorecer o melhor desempenho de produção.

\section{METODOLOGIA}

\section{1 Água Residual da Aquicultura, Estirpe de Algas e Meio de Cultura}

O efluente aquícola foi gentilmente cedido pela Bahia Pesca S/A, localizada na Fazenda Joanes II, em Camaçari - Bahia (Nordeste do Brasil, 1241'51"S e longitude $38^{\circ} 19^{\prime} 27^{\prime \prime W}$ ). A cepa de Spirulina foi isolada da Lagoa Mangueira, no sul do Brasil (latitude $32^{\circ} 32^{\prime} 05$ "S e longitude $33^{\circ} 31^{\prime} 57^{\prime \prime} \mathrm{W}$ ) e mantida em meio de Zarrouk [4]. As cianobactérias Spirulina sp. (LEB 18) foi obtida da Coleção de Cultura do Laboratório de Engenharia Bioquímica da Universidade Federal do Rio Grande (FURG).

\subsection{Preparação do Inoculo e Fluxograma Experimental}

A água residual do cultivo de tilápia (Oreochomis niloticus) foi primeiramente suplementada com diferentes porcentagens do meio sintético de Zarrouk (ZM) $\mathrm{NaHCO}_{3} 16.8 \mathrm{~g} / \mathrm{L} ; \mathrm{NaNO}_{3} 2.5 \mathrm{~g} / \mathrm{L} ; \mathrm{NaCl} 1.0 \mathrm{~g} / \mathrm{L} ; \mathrm{K}_{2} \mathrm{HPO}_{4} 0.5 \mathrm{~g} / \mathrm{L} ; \mathrm{K}_{2} \mathrm{SO}_{4} 1.0 \mathrm{~g} / \mathrm{L} ;$ 
$\mathrm{MgSO}_{4} \cdot 7 \mathrm{H}_{2} \mathrm{O} 0.2 \mathrm{~g} / \mathrm{L} ; \mathrm{CaCl}_{2} 0.031 \mathrm{~g} / \mathrm{L} ; \mathrm{Na}_{2} \mathrm{EDTA} 0.08 \mathrm{~g} / \mathrm{L} ; \mathrm{FeSO}_{4} \cdot 7 \mathrm{H}_{2} \mathrm{O} 0.01 \mathrm{~g} / \mathrm{L}$ (Costa et al. 2004). Os experimentos foram realizados apenas com a água residual da aquicultura (T-0) e com os respectivos tratamentos 25,50 e $75 \%$ dos nutrientes totais de Zarrouk (T-25, T-50 e T-75) seguindo proporções similares ao adotado por Kuo et al. (2015) e Daneshvar et al. (2018). Após a suplementação, cada tratamento foi inoculado com a Spirulina sp. (LEB 18) (no fim da fase exponencial). O crescimento foi monitorado por sete 7 dias, após o período a biomassa foi coletada para avaliar a produção. [5,6]

\subsection{Desenho Experimental}

A biomassa da Spirulina sp. (LEB18) foi obtida a partir de um tanque de pista $(210 \mathrm{~L})$ em cultura contínua (1,0 biomassa g/L) mantido com o meio Zarrouk. Os diferentes ensaios foram preparados em Erlenmeyer com um volume de trabalho de $1 \mathrm{~L}$. A água residual de aquicultura $(800 \mathrm{ml})$ foi inoculada com a Spirulina sp. (LEB18) com concentração de $0,3 \mathrm{~g} / \mathrm{L}$ de biomassa viva. Os experimentos seguiram a metodologia proposta por Kuo et al. (2015) e Daneshvar et al. (2018)[5,6]

Os tratamentos experimentais (T-25, T-50 e T-75) foram preparados com o fornecimento de fotobiorreatores de $1 \mathrm{~L}$ (água de aquacultura/inóculo) com 25, 50 e $75 \%$ do total de nutrientes médios de Zarrouk individualmente. O T-0 corresponde ao efluente aquícola sem suplementação de nutrientes e o controle ao meio sintético Zarrouk. A incubação foi realizada durante 7 dias a $30^{\circ} \mathrm{C}$ com fotoperíodo de 12 horas claro/escuro. $\mathrm{O}$ ar foi fornecido a todas as culturas e lâmpadas tubulares fluorescentes com uma intensidade de luz de 41,6 $\mu$ molphotons $\mathrm{m}^{-2} \mathrm{~s}^{-1}$ foram usadas como fonte de energia.

\subsection{Parâmetros de Crescimento e Determinação de pH}

A concentração da biomassa de Spirulina sp. (LEB18) foi determinada diariamente, a partir da densidade óptica das culturas em espectrofotômetro (PerkinElmer Lambda 35 UV/VIS) no comprimento de onda de 670nm em ambos tipos de reatores. Uma curva padrão foi construída antes dos experimentos, relacionando a densidade óptica do inoculo com sua massa seca [7]. Parâmetros de crescimento foram determinados para cada dia de cultivo. A produtividade foi obtida pela Equação 1 , onde $X_{t}$ é a concentração de biomassa $\left(\mathrm{gL}^{-1}\right)$, $\mathrm{t}(\mathrm{d})$ tempo e $\mathrm{X}_{0}$ a concentração de biomassa $\left(\mathrm{gL}^{-1}\right)$ no tempo to $(\mathrm{d})$ inicial.

$$
P_{x}=\frac{X_{t}-X_{0}}{t-t_{0}}
$$

A taxa de crescimento específica ( $\mu$ esp) foi obtida a partir da regressão linear na fase log de cultivo. $\mathrm{O} \mathrm{pH}$ também foi monitorado diariamente com o auxílio de um medidor digital de $\mathrm{pH}$. 


\section{RESULTADOS E DISCUSSÃO}

Os resultados mostraram que as águas residuais de aquicultura suplementadas com 25 e $50 \%$ de ingredientes Zarrouk (T-25 e T-50) favoreceram uma redução da fase de adaptação para a Spirulina sp. (LEB18) (Figura 1). O crescimento após um dia de cultivo revela que as microalgas se adaptaram bem às novas condições ambientais [4]. O potencial de fitorremediação das algas está intimamente ligado ao seu crescimento, maior crescimento de algas promoverá melhor remoção de nutrientes dos meios de cultivo [1]. Resultados semelhantes ao presente estudo foram relatados por Lu et al. (2019) usando Zeólita para mitigar a toxicidade de amônia em águas residuárias para a produção de Spirulina com valor agregado, não houve fase de adaptação em tratamentos contendo 42,5 e $127,5 \mathrm{mg} / \mathrm{L}_{\text {de }} \mathrm{NH}_{4+}$. [8]

Figura 1. Monitoramento da produção de biomassa durante o cultivo de Spirulina sp. (LEB18) em 100\% de água residual aquícola suplementada com diferentes concentrações de Zarrouk (T-75, T-50, T-25 e T-0) por 7 dias. Média \pm desvio padrão. Letras sobrepostas equivalentes (a) indicam que não houve diferença significativa entre os valores do nível de confiança de $95 \%$.

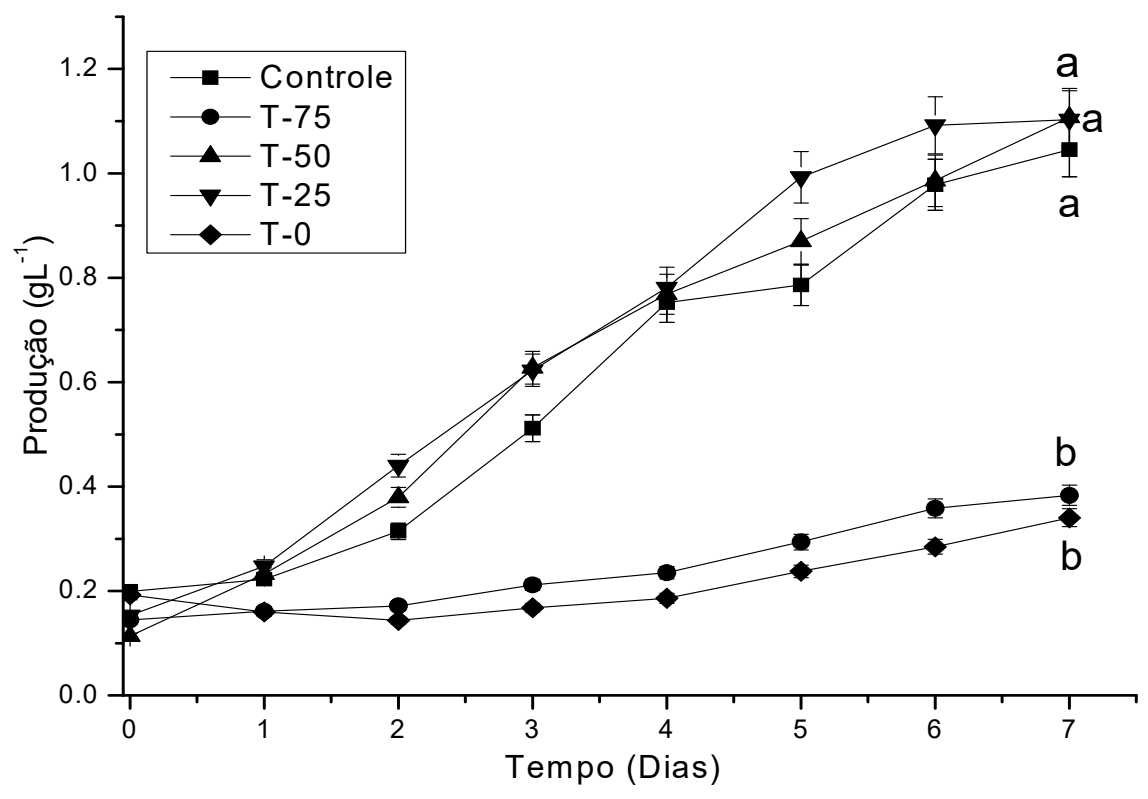

Comparações estatísticas mostraram que a produção total obtida com T-25, T50 e o controle com meio sintético (T-C) foram semelhantes após 7 dias de cultivo (Figura 1). Apesar do fato de que a taxa de produção de T-50 foi significativamente maior (Tabela 1) nos primeiros 6 dias, o tratamento T-25 apresentou maior produção de biomassa $\left(1,10 \mathrm{gL}^{-1}\right)$ (Tabela 1). Zhou et al. (2017) relatou que a Spirulina sp. cultivada em água de esgoto misturado com água do mar (1: 0.7: 3.5: 5 e 3: 7, respectivamente) geraram um rendimento máximo de $0.81 \mathrm{gL}^{-1}(5: 5)$ dentro de 10 dias. Por outro lado, Zhang et al. (2019) avaliaram o crescimento de Chlorella sorokiniana em águas residuais de maricultura (MW) modificadas com resíduo de glutamato 
monossódico (RGMS) ou $\mathrm{NaNO}_{3}$ e $\mathrm{K}_{2} \mathrm{HPO}_{4}$. A maior produção atingiu $0,5 \mathrm{gL}^{-1}$ na suplementação de água residual com RGMS. O cultivo foi realizado em Erlenmeyer de 1 litro por 10 dias, os resultados encontrados foram inferiores ao presente estudo, os autores atribuíram ao estresse no ambiente. [7,8]

Tabela 1. Parâmetros cinéticos de produção $\left(\mathrm{gL}^{-1}\right)$, taxa de produção $\left(\mathrm{gL}^{-1} \mathrm{~d}^{-1}\right)$, crescimento específico $\mu_{\text {esp. }}\left(\mathrm{d}^{-1}\right)$ e $\mathrm{pH}$ da Spirulina sp. (LEB18) cultivada em diferentes concentrações de Zarrouk (Controle, T-75, T-50, T-25, T-0) em 100\% da água residual da aquicultura. Média \pm desvio padrão. Letras equivalentes sobrepostas (a-c) no mesmo tipo de coluna indicam que não houve diferença significativa entre os valores do nível de confiança de $95 \%$.

\begin{tabular}{ccccc}
\hline $\begin{array}{c}\text { Tratamento } \\
(\%)\end{array}$ & $\begin{array}{c}\text { Produção } \\
\left(\mathbf{g L}^{-1}\right)\end{array}$ & $\begin{array}{c}\text { Taxa de } \\
\text { Produção } \\
\mathbf{P}\left(\mathbf{g L}^{-1} \mathbf{d}^{-1}\right)\end{array}$ & $\boldsymbol{\mu}_{\text {esp. }}\left(\mathbf{d}^{-1}\right)$ & pH \\
\hline Controle & $1.05 \pm 0.08^{\mathrm{a}}$ & $0.04 \pm 0.01^{\mathrm{ab}}$ & $0.25 \pm 0.01^{\mathrm{a}}$ & $10.30 \pm 0.02^{\mathrm{a}}$ \\
T-75 & $0.39 \pm 0.01^{\mathrm{b}}$ & $0.04 \pm 0.02^{\mathrm{bc}}$ & $0.25 \pm 0.01^{\mathrm{a}}$ & $11.88 \pm 0.01^{\mathrm{c}}$ \\
T-50 & $1.02 \pm 0.11^{\mathrm{a}}$ & $0.12 \pm 0.04^{\mathrm{a}}$ & $0.33 \pm 0.03^{\mathrm{b}}$ & $10.39 \pm 0.01^{\mathrm{a}}$ \\
T-25 & $1.10 \pm 0.26^{\mathrm{a}}$ & $0.20 \pm 0.05^{\mathrm{ab}}$ & $0.27 \pm 0.04^{\mathrm{a}}$ & $11.06 \pm 0.03^{\mathrm{b}}$ \\
T-0 & $0.34 \pm 0.04^{\mathrm{b}}$ & $0.03 \pm 0.01^{\mathrm{c}}$ & $0.18 \pm 0.11^{\mathrm{c}}$ & $10.22 \pm 0.05^{\mathrm{a}}$ \\
\hline
\end{tabular}

A amônia não foi detectada no efluente aquícola utilizado nesta pesquisa isto pode ter influenciado os resultados citados. Porque altas concentrações de amônio reduziria a produção de biomassa ou até mesmo causaria a falha do cultivo de algas, devido ao aumento do estresse oxidativo intracelular ou perturbando o sistema de transporte de elétrons [9]. Ramsundar et al. (2017) relataram o uso de águas residuais de aquicultura suplementadas com nitrogênio para o cultivo de Chlorella sorokiniana. A maior taxa de produção $\left(0,49 \mathrm{gL}^{-1} \mathrm{~d}^{-1}\right)$ foi observada com uma suplementação de nitrogênio de $400 \mathrm{mgL}^{-1}$. [10]

A taxa de crescimento e disponibilidade de nutrientes são dependentes dos valores de $\mathrm{pH}$ [3]. Variações nos valores de $\mathrm{pH}$ não foram significativas durante o cultivo e permaneceram nos níveis muito alcalinos durante todo o período de incubação. Os valores iniciais de pH foram 8-9 e aumentaram para 10-11, podendo ser atribuído a esterilização, que decompõe algumas substâncias devido às altas temperaturas produzirem $\mathrm{OH}$ - portanto, aumentando o $\mathrm{pH}$ [11]. Existe uma vantagem significativa de cultivar Spirulina sp. em valores altos de pH. Evita a contaminação ou o desenvolvimento de cultivos mistos de algas. Contaminação por outras microalgas em cultivos de Spirulina sp. culturas podem diminuir a produção de biomassa em aproxidamente $75 \%$. [11]

Alguns autores relataram que a alta concentração de matéria orgânica pode inibir o crescimento de microalgas [11], justificando o baixo crescimento em T-0 e o baixo rendimento em T-75. Porque a concentração de sólidos dissolvidos totais, matéria orgânica e o meio Zarrouk (rico em sais) adicionado $75 \%$ podem afetar a disponibilidade de nutrientes na água. Pois alguns processos químicos, como a precipitação química, podem remover parte do nitrogênio e do fósforo das águas residuais. [13] 
O maior crescimento específico $\left(\mu_{\text {esp. }} \mathrm{d}^{-1}\right)$ foi relatado no tratamento T-50 $(0,33$ $\left.\mu_{\text {esp. }} \mathrm{d}^{-1}\right)$ e T-25 $\left(0,27 \mu_{\text {esp. }} \mathrm{d}^{-1}\right)$. Resultados semelhantes ao presente estudo foram encontrados por Luo et al. (2016) em Coelastrella sp. QY01 cultivada em efluente de suinocultura tratado com diluição de 20 a 100\%, na fase exponencial da cultura batelada, as taxas de crescimento específicas variaram de 0,269 a 0,325 dia $^{-1}$. De acordo com os resultados dos parâmetros cinéticos, os tratamentos (T-25 e T-50) podem ser aplicados no sistema de recirculação de aquicultura (RAS) segundo Egloff et al. (2018) esses sistemas limitam as densidades atingíveis de biomassa de microalgas a 1-2 $\mathrm{g} \mathrm{dw}^{-1}$, depois que os níveis de nitrogênio estão esgotados e assumindo que o nitrogênio é o nutriente limitante e ocorre a uma concentração de aproximadamente $100 \mathrm{mgL}^{-1} \mathrm{em}$ um RAS. $[13,14]$

\section{CONCLUSÃO}

O estudo demonstrou o desempenho de crescimento da Spirulina sp. (LEB18) em águas residuais de aquicultura suplementadas com quantidades distintas de nutrientes. Os melhores resultados foram obtidos usando $25 \%$ dos ingredientes gerais descritos no meio sintético Zarrouk. Assim, a Spirulina sp. (LEB18) cultivada em água residual do cultivo de Oreochomis niloticus suplementado com $25 \%$ de Zarrouk, representa uma alternativa sustentável para obtenção de biomassa utilizando água residual da aquicultura.

\section{Agradecimentos}

Todos os autores agradecem o apoio da FAPESB - Fundação de Amparo à Pesquisa da Bahia no projeto CNPQ (400710 / 2014-5), MCTIC (Ministério da Ciência e Tecnologia da Informação e Comunicação) - Brasil e Bahia Pesca.

\section{REFERÊNCIAS}

${ }^{1}$ Wuang, S. C. Khin, M. C. Chua, P. Q. D. Luo, Y. D. (2016). Use of Spirulina biomass produced from treatment of aquaculture wastewater as agricultural fertilizers. Algal Res, 15, 59-64.

${ }^{2}$ Ferreira, J. G. L. Falconer, J. Kittiwanich, L. Ross, C. Saurel, K. Wellman, C. B. Z. P. Suvanacha. (2015). Analysis of production and environmental effects of Nile tilapia and white shrimp culture in Thailand. Aquac. Res 447, 23-36.

${ }^{3}$ Salama, E. Byong-Hun, J. B. Chang, W. S. Lee, S. Roh, H. Yang, I. (2017). Interactive effect of indole-3-acetic acid and diethyl aminoethyl hexanoate on the growth and fatty acid content of some microalgae for biodiesel production. J. Clean. Prod, 168, 10171024. 
${ }^{4}$ Costa, J.A.V. Colla, L.M. Duarte Filho, P. Kabke, K. Weber, A. (2004). Modelling of Spirulina platensis growth in fresh water using response surface methodology. World J Ind Microbiol Biotechnol , 18, 603-607.

5 Kuo, C. Chen, T. Lin, T. Kao, C. Lai, J. Chang, J. Lin, C. (2015). Cultivation of Chlorella sp., GD using piggery wastewater for biomass and lipid production. Bioresour. Technol, 194, 326-333.

6 Daneshvar, E. Antikainen, L. Koutrac, E. Kornarosc, M. Bhatnagara, A. (2018). Investigation on the feasibility of Chlorella vulgaris cultivation in a mixture of pulp and aquaculture effluents: Treatment of wastewater and lipid extraction. Bioresour. Technol, 255, 104-110.

7 Zhou, W. Yanting, L. Goa Y. Haixia Z. (2017). Nutrients removal and recovery from saline wastewater by Spirulina platensis. Bioresour. Technol, 245, 10-17.

8 Xu, X. Wang, J. Zhang, T. Dao, G. Wu, G. Hu, H. (2017). Attached microalgae cultivation and nutrients removal in a novel capillary-driven photo-bio film reactor. Algal Res, 32, 198-205.

9 Lu, W. Alam, M.A. Luo, W. Asmatulu, E. (2019). Integrating Spirulina platensis cultivation and aerobic composting exhaust for carbon mitigation and biomass production. Bioresour. Technol, 271, 59-65.

10 Ramsundar, P. Abhishek G, Singh P. Pillay, K. Bux, F. (2017). Evaluation of wate activated sludge as a potential nutrient source for cultivation of Chlorella sorokiniana. Algal Res, 28,108-117.

11 Yang, F. Xiang, W. Fan, J. Wu, H. Li, T. Long, L. (2016). High pH-induced flocculation of marine Chlorella sp. for biofuel production. J Appl Phycol, 28, 747-756.

12 Cheirsilp, B and Torpee, S. (2012). Enhanced growth and lipid production of microalgae under mixotrophic culture condition: effect of light intensity, glucose concentration and fed-batch cultivation. Bioresour. Technol, 110, 510-516.

${ }^{13}$ Luo, L. He, H. Yang, C. Wen, S. Zeng, G. Wu, M. Zhou, Z. Lou, W. (2016). Nutrient removal and lipid production by Coelastrella $s p$. in anaerobically and aerobically treated swine wastewater. Bioresour. Technol, 216, 135-141.

14 Egloff, S. Tschudi, F. Schmautz, Z. Refardt, D. (2018). High-density cultivation of microalgae continuously fed with unfiltered water from a recirculating aquaculture system. Algal Res, 34, 68-74. 